\title{
Regard sur l'expérience de vivre avec le cancer : Le cancer comme occasion de changement chez des personnes âgées de 60 à 73 ans*
}

\author{
Joanie Gagnon, ${ }^{1}$ Anouk Utzschneider, ${ }^{1}$ and André Doyon²
}

\begin{abstract}
It is possible for individuals with a diagnosis of cancer to see the illness as an opportunity to change and to make certain decisions that could improve their chances of recovery. We aimed, through qualitative research, to understand the experience of cancer in older adults through new behaviors and habits, intentions to change and maintenance of old behaviors and habits in the context of the disease. Our participants were 10 people aged 60 to 73 with a diagnosis of cancer. The majority of participants expressed intentions to change or adopted new behaviors or habits. Relatives had very little influence on participant's choices and participants did not seek information on cancer or ways to contribute to healing. However, they were very receptive to the recommendations made by physicians.

\section{RÉSUMÉ}

Il est possible pour les individus ayant reçu un diagnostic de cancer de concevoir la maladie comme une occasion de changement et de prendre certaines décisions qui pourraient améliorer leurs chances de guérison. Nous avons voulu comprendre l'expérience du cancer chez les personnes âgées à travers les agirs nouveaux, les intentions d'agir et le maintien des agirs antérieurs dans le contexte de la maladie à l'aide d'une recherche qualitative. Le groupe de participants était composé de 10 personnes âgées de 60 et 73 ans atteintes de cancer. La majorité des participants ont manifesté des intentions d'agir ou ont adopté de nouveaux agirs. L'entourage avait très peu d'influence sur les choix et les participants n'ont pas cherché à obtenir par eux-mêmes de l'information sur le cancer ou sur des façons de contribuer à la guérison. Ils ont toutefois été très réceptifs aux recommandations des médecins.
\end{abstract}

1 Centre de formation médicale du Nouveau-Brunswick, Université de Moncton, campus de Moncton, Moncton

2 Faculté des sciences de l'éducation, Département d'enseignement au primaire et de psychopédagogie, Université de Moncton, campus de Moncton, Moncton

* Nous tenons à remercier la Fondation de l'Arbre de l'espoir et le Réseau de santé Vitalité pour leur contribution financière à ce projet. Nous remercions également le personnel de l'Auberge Mgr.-Henri-Cormier pour leur accueil et Dre Diane Pruneau, professeure à l'Université de Moncton, pour sa contribution intellectuelle.

Manuscript received: / manuscrit reçu : 23/06/16

Manuscript accepted: / manuscrit accepté : 11/10/17

Keywords: cancer, changes, older adults, intentions to change, new behaviors and habits, qualitative research

Mots-clés : cancer, changements, adultes âgés, intentions d'agir, agirs nouveaux, recherche qualitative

Correspondence and requests for reprints should be sent to / La correspondance et les demandes de tirés-à-part doivent être adressées à:

Joanie Gagnon, B.Psy. (Candidate à la maîtrise en travail social)

École de travail social

Université de Moncton, campus de Moncton

Moncton, NB E1A 3E9

(ejg8518@umoncton.ca)

Canadian Journal on Aging / La Revue canadienne du vieillissement 37 (3) : 281-293 (2018)

Copyright (C) Canadian Association on Gerontology 2018. This is an Open Access article, distributed under the terms of the

Creative Commons Attribution licence (http://creativecommons.org/licenses/by/4.0/), which permits unrestricted re-use,

distribution, and reproduction in any medium, provided the original work is properly cited. 
Anouk Utzschneider, PhD (auteur de correspondance) Centre de formation médicale du Nouveau-Brunswick Université de Moncton, campus de Moncton

Moncton, NB, E1A 3E9 Canada

(Anouk.utzschneider@umoncton.ca)

\section{Introduction}

On constate dans les sociétés occidentales une hausse importante du nombre de cas de cancer, et le vieillissement de la population serait la principale cause de cette augmentation au Canada (Société canadienne du cancer, 2016). D'ici 2028-2032, on estime que le nombre annuel moyen de nouveaux cas de cancer augmentera de $79 \%$ par rapport à 2003-2007. Environ 45\% des cancers diagnostiqués en 2017 l'ont été chez des personnes âgées de 70 ans et plus, et $28 \%$ chez des personnes âgées de 60 à 69 ans (Société canadienne du cancer, 2017).

Par ailleurs, un nombre croissant de recherches nous indiquent que les habitudes de vie ont un impact sur l'évolution du cancer. D'après les chiffres avancés par la Société américaine du cancer (2016), environ 75\% des cancers seraient le produit de styles de vie (ex. alimentation, activité physique, consommation d'alcool, tabac...) ou d'un environnement malsain (ex : environnement de vie pollué, exposition professionnelle à des carcinogènes...). Or, ces variables peuvent en partie être modifiées. Plusieurs études (ex. Doraia \& Aggarwal, 2004; Heidermann et al., 2008; Krukowski \& Smith West, 2010) suggèrent que certains aliments inhibent les agents cancérigènes. Du point de vue de l'activité physique, une méta-analyse (Barbaric, Brookes, Moore, \& Cheifetz, 2010) concernant les effets de l'activité physique sur la survie des personnes ayant reçu un diagnostic de cancer a permis de constater que les individus pratiquant une activité physique plus soutenue risquaient moins de mourir du cancer. D'autres recherches ont permis de mettre en évidence, par exemple, les liens entre la pollution de l'air et l'augmentation de la mortalité due au cancer du poumon (ex: Arden Pope III et al., 2002; Vineis, Hoek, Krzyzanowski, VignaTaglianti, \& Veglia, 2007). Comme les niveaux de pollution de l'air peuvent varier sur de faibles distances (Blanchard, Tanenbaum, \& Hidy, 2014), les individus peuvent diminuer leur niveau d'exposition à la pollution en changeant de ville ou même de quartier. Dans une société où l'information sur la santé est plus disponible que jamais, il est donc possible pour les individus ayant reçu un diagnostic de cancer de concevoir le cancer comme une occasion de changement et de prendre certaines décisions qui pourraient améliorer les chances de guérison. Les changements peuvent également être de l'ordre d'une redéfinition de la vision globale de la vie, des relations et des priorités (Halldorsdottir \& Hamrin, 1996; Esteves, Roxo, \& da Conceicao Saraiva, 2015).
Thomé, Esbensen, Dykes, et Hallberg (2004) soulignent que plusieurs recherches ont été effectuées sur le cancer, mais que très peu concernent l'expérience de vie des gens plus âgés qui en sont atteints. Comprendre l'expérience vécue implique, selon Van Manen (1997), une conscience immédiate et réflexive de la personne sur sa propre vie. Thomé et al. (2004) ont cherché à comprendre le sens du cancer comme expérience vécue à un âge avancé. Leurs résultats ont indiqué que tant qu'aucun effet secondaire n'était présent, l'expérience de vie des gens n'était pas affectée. Par contre, ces effets secondaires, lorsqu'ils étaient présents, influençaient leur vie de façon négative en amenant une redéfinition des rôles, un rétrécissement de l'espace de vie et une grande fatigue. Selon les auteurs, ces difficultés étaient exacerbées par l'âge avancé des participants. Afin d'améliorer leur expérience, les participants cherchaient à entretenir de bonnes relations avec leur entourage. Les auteurs soulignent l'importance des relations avec le personnel médical et les médecins puisqu'une mauvaise attitude de ces derniers rendrait l'expérience de vivre avec le cancer plus négative. Les participants ont d'ailleurs souligné qu'ils avaient souvent l'impression d'être moins considérés par le personnel médical et les médecins en raison de leur âge.

Selon Towsley, Beck, et Watkins (2007), le processus d'adaptation à la maladie est dynamique et il est influencé par l'expérience de vie antérieure de la personne âgée, son attitude, le sens du soi, les facteurs positifs et négatifs qui interviennent dans le processus ainsi que les stratégies d'adaptation adoptées par les individus. Ces stratégies sont par exemple : la modification des activités, le support social, l'acceptation réfléchie, la spiritualité, le dépassement des attentes, le maintien des routines ainsi que le relativisme interne et externe (soit de comparer leur expérience à des expériences antérieures ou à celles d'autres individus dans des situations pires que la leur). Cette dernière stratégie est plus fréquente chez les personnes âgées étant donné que leurs expériences de vie antérieures les ont, dans la plupart des cas, déjà fait surmonter d'autres difficultés.

Towsley et al. (2007) soulignent qu'en plus des facteurs sociaux, des facteurs financiers et physiques influencent positivement ou négativement le choix des stratégies d'adaptation utilisées par les individus atteints de cancer. Par exemple, des symptômes faibles, un bon suivi médical et une certaine aisance financière aideraient les individus à atteindre un certain équilibre. Selon le modèle 
développé par les auteurs, l'attitude serait toutefois le facteur le plus fondamental.

Le cancer peut, en raison du stress vécu, éventuellement se transformer en apprentissage (Cordova \& Andrykowski, 2003). Des changements positifs dans la vie des patients sont souvent observés suite aux traitements. Ils ont tendance à redéfinir et repenser leurs objectifs de vie et à apprécier davantage leur vie en développant des nouvelles stratégies d'adaptation. Même si certains comportements nouveaux s'avèrent négatifs, plusieurs patients et survivants adoptent des changements positifs comme des relations conjugales plus saines, une plus grande foi, une attitude plus positive envers la vie ainsi que de meilleures relations avec les membres de leur famille et avec leurs amis (Andrykowski, Brady, \& Hunt, 1993).

Ainsi, plusieurs études ont été faites au sujet de l'expérience des gens atteints de cancer, notamment en lien avec les changements qu'elle génère et les stratégies d'adaptation mises en place. La plupart de ces études ciblent toutefois les survivants ou les personnes en rémission. Peu de recherches sont effectuées auprès des gens atteints de cancer et en traitement. Aussi, rares sont celles qui concernent seulement les personnes plus âgées.

Notre intérêt de recherche se situe plus particulièrement au niveau des occasions de changement dans l'expérience du cancer chez les personnes plus âgées qui étaient en traitement au moment des entretiens. Nous avons voulu comprendre l'expérience du cancer chez ces personnes à travers les agirs nouveaux, les intentions d'agir et le maintien des agirs antérieurs dans le contexte de la maladie. Ce questionnement sert de fil conducteur à cette contribution, laquelle poursuit trois objectifs, à savoir :

- Identifier et comprendre les intentions d'agir liées à l'expérience de la maladie;

- Identifier et comprendre les agirs nouveaux liés à l'expérience de la maladie;

- Identifier et comprendre le maintien des agirs antérieurs dans le contexte de la maladie.

\section{Cadre conceptuel}

Selon les résultats d'une recherche menée par Doyon, Legault, \& Paré (2008) auprès d'un groupe d'enseignants, le processus de transformation intérieure que vivent les individus lorsqu'ils font face à une situation imprévue les amène à formuler des intentions d'agir et aussi à entreprendre des agirs nouveaux. L'intention d'agir et l'agir nouveau permettent d'expliquer plusieurs phénomènes qui se présentent dans les processus de changement ou le maintien de certains agirs antérieurs à la maladie. Ce processus de transformation intérieure peut être transposé à différentes situations de la vie et semble ainsi constituer un cadre approprié à l'étude des processus de changement ou de maintien des agirs antérieurs chez des personnes âgées vivant une situation de santé souvent inattendue. D'autres auteurs ont également parlé d'intentions et d'agirs nouveaux dans plusieurs contextes, par exemple celui du support offert par la famille (Senden et al., 2015), des habitudes alimentaires (Hopkinson, 2007) et de l'attitude des survivants âgés envers leur vie suite au cancer (Towsley, Beck and Watkins, 2007).

\section{L'intention d'agir}

L'intention correspond à la vision claire d'un objectif à atteindre (Assagioli, 1989). Elle apparait comme un désir d'être ou d'agir autrement. L'intention peut survenir à la suite d'une prise de conscience ou encore lorsque l'entourage de la personne lui laisse entrevoir son opinion ou lui donne de la rétroaction (Doyon et al., 2008).

Senden et al. (2015) pour leur part, expliquent que les gens atteints de cancer qui sont en traitement ont des intentions pour leur futur. Certains ont pour but de retourner travailler, d'autres mentionnent que leur objectif est de reprendre leurs activités habituelles lorsque leurs traitements seront terminés. Doyon et al. (2008) soulignent que le fait d'avoir l'intention et le désir d'adopter de nouveaux comportements n'est pas suffisant. La personne doit passer à l'action et entreprendre un agir nouveau.

\section{L'agir nouveau}

Lié à des représentations et à des intentions nouvelles, l'agir nouveau consiste essentiellement à sortir de la répétition, à faire autrement. Dans leur étude, Doyon et al. (2008) ont souligné deux façons dont les personnes agissent : spontanément et de façon délibérée. Dans l'agir nouveau spontané, la personne se voit tout d'un coup en train d'agir de manière différente. Dans l'agir nouveau délibéré, la personne choisit consciemment de manifester un comportement qu'elle avait résolu d'adopter. Doyon et al. (2008) reprennent les propos de Garneau et Larivey (1979) en mentionnant que le fait de changer des comportements se fait le plus souvent par étapes successives et non d'un seul coup.

Des chercheurs ont remarqué certains agirs nouveaux dans les habitudes de vie et dans les comportements des gens lorsqu'ils ont le cancer. Par exemple, Hopkinson (2007) a remarqué des changements dans l'alimentation des personnes atteintes de cancer dès le début de leur maladie. Leurs résultats indiquent que la quantité et la qualité de la nourriture consommée par les participants âgés s'avérait être meilleure depuis qu'ils avaient reçu leur diagnostic de cancer. Pour leur part, Senden et al. (2015) ont trouvé que la présence du cancer occasionne, 
dans la famille de la personne âgée atteinte, un changement dans les rôles et dans les tâches entre les membres de cette famille.

Certains auteurs remarquent aussi que les changements ne sont pas toujours présents. Les résultats d'une étude effectuée auprès de 55 survivants du cancer âgés entre 65 et 81 ans par Towsley et al. (2007) indiquent que plusieurs retournent à leurs vieilles habitudes, à leur routine, continuent de travailler et poursuivent leur bénévolat, même durant leurs traitements. Cependant, plusieurs ont admis avoir été obligés d'établir des priorités parmi ces activités.

Selon plusieurs auteurs (Fromm, Andrykowski, \& Hunt, 1996; Petrie, Buick, Weinman, \& Booth, 1999), environ 60 à $90 \%$ des gens atteints de cancer adoptent des changements positifs dans leur vie. Il semblerait que ces changements positifs soient influencés par le contrôle que ces derniers ont sur eux-mêmes, sur leurs décisions et sur leur vie (Komura \& Hegarty, 2006). Selon ces auteurs, les patients qui ont un haut niveau d'auto-efficacité ont plus de facilité à s'adapter à leur cancer et ont moins tendance à avoir des résultats psychologiques négatifs.

\section{Méthodologie}

La recherche qualitative proposée emprunte une approche inductive modérée. Selon Karsenti \& SavoieZajc (2011), l'approche inductive modérée consiste à reconnaître l'influence du cadre théorique par la définition des concepts étudiés. Les différentes dimensions qui ont servi à qualifier les concepts ont cependant été mises de côté pour le temps de l'analyse afin de laisser émerger les catégories. Ces dernières se sont toutefois insérées de façon naturelle à l'intérieur des concepts globaux décrits dans le cadre conceptuel. Nous avons tout de même légèrement reformulé ces concepts afin qu'ils reflètent mieux nos résultats de recherche.

\section{Participants}

\section{Population}

Les participants de notre recherche étaient 10 personnes ( 5 hommes et 5 femmes) âgés de 60 et 73 ans atteintes de cancer qui séjournaient à l'Auberge Mgr-HenriCormier. Cette auberge, située à Moncton au NouveauBrunswick, héberge les patients qui vivent à plus de $100 \mathrm{~km}$ de l'hôpital où sont offerts les traitements d'oncologie. Les cinq hommes étaient atteints du cancer de la prostate, trois femmes étaient atteintes du cancer du sein, une était atteinte du cancer du col de l'utérus et la dernière était atteinte du cancer de la vessie. Chez les femmes, trois étaient veuves, une était divorcée et une était célibataire mais vivait avec sa mère et sa sœur. Cette dernière était la seule à ne pas avoir eu d'enfants. Trois avaient terminé leur primaire et deux leur secondaire. Toutes les femmes étaient à la retraite mais deux étaient très actives comme bénévoles. Chez les hommes, tous étaient mariés et avaient des enfants maintenant adultes. Deux avaient terminé leur primaire et trois leur secondaire. Trois des hommes étaient toujours sur le marché du travail et deux à la retraite. Aucune distinction n'a été faite en ce qui a trait au type de cancer pour lequel les individus recevaient des traitements ni au stade auquel se situait leur cancer. Ainsi, toutes les personnes volontaires de 60 ans et plus qui se sentaient capables de participer au projet et dont la durée de séjour était d'au moins 5 semaines étaient invitées à prendre part à la recherche. Il s'agissait donc d'un échantillonnage de convenance. Le hasard a fait qu'aucune personne de plus de 73 ans ne s'est présentée pendant la période de recrutement. Nos participants étaient donc un groupe de « jeunes personnes âgées ». La durée de 5 semaines était requise afin de permettre la tenue de deux entrevues en laissant un certain temps de réflexion entre les deux. Les participants avaient le choix de faire l'entrevue en français ou en anglais. Ils avaient également l'option de faire l'entrevue avec un homme ou une femme. Deux entrevues ont été conduites en anglais, et tous ont affirmé être à l'aise que l'entrevue soit conduite par une femme. Les entrevues ont donc toute été menées par la même chercheure de l'équipe.

Bien qu'il ne s'agissait pas d'un critère de sélection, les participants recrutés se sont avérés être tous peu scolarisés (formation de niveau primaire ou secondaire). La province du Nouveau-Brunswick compte en effet des taux élevés de pauvreté et des niveaux de scolarité faibles en comparaison avec les moyennes canadiennes (Statistique Canada, s.d.). La plupart des participants étaient toutefois encore actifs professionnellement ou comme bénévoles.

\section{Identification et recrutement des participants}

L'identification des participants potentiels répondant aux critères de sélection a été faite dans un premier temps par la gestionnaire de l'Auberge Mgr-HenriCormier. Cette dernière accueille les nouveaux résidants et était ainsi en mesure d'effectuer une présélection des participants potentiels auxquels elle parlait brièvement de la recherche. Si une personne semblait intéressée, elle déterminait avec elle un temps qui lui convenait afin qu'un membre de notre équipe de recherche la rencontre à l'Auberge pour lui fournir davantage d'explications sur le projet. Nous avons recruté des participants jusqu'à l'atteinte de la saturation lors de l'analyse préliminaire parallèle des données.

\section{Méthode de collecte de données}

L'entrevue semi-dirigée, conduite à deux reprises auprès des participants, a été notre outil de collecte de données. 
La première entrevue était conduite en moyenne une semaine après que la personne ait accepté de prendre part au projet. Lors de cette entrevue, la chercheure et le participant avaient l'occasion de faire connaissance afin qu'un lien de confiance puisse être établi. Par la suite, les thèmes suivants ont été abordés en entrevues :

- L'expérience globale du cancer (type de cancer, récidives, traitements, effets secondaires, signification...)

- Le contexte de l'annonce du diagnostic et la réaction à l'annonce

- Les changements apportés ou planifiés depuis l'annonce du diagnostic

- Le rôle de la famille, de l'entourage, du personnel médical et de soutien et des autres patients de l'Auberge dans les changements apportés ou prévus

- Le rôle des sources d'information sur la santé (livres, revues, ateliers, émissions, internet, dépliants, etc) dans les changements apportés ou prévus

- Les éléments de leur vie (contexte, personnalité, vécu...) qui aident à passer à travers l'expérience du cancer

- Les éléments de leur vie (contexte, personnalité, vécu...) qui rendent plus difficile l'expérience du cancer

Au cours des semaines subséquentes, les participants étaient invités à porter attention aux évènements ou informations reçues qui influençaient leurs décisions en rapport avec leur santé. La deuxième entrevue s'est tenue environ un mois après la première. Lors de cette entrevue, la chercheure a fait un retour sur les observations, réflexions et décisions des participants. Elle a aussi posé des questions concernant les changements relatifs à leur santé depuis la première entrevue afin de mieux situer l'évolution de leur expérience.

Chaque entrevue a duré en moyenne une heure et a été réalisée à un moment proposé par le participant. Toutes les entrevues ont été réalisées à l'Auberge et en toute confidentialité. Les entrevues ont été enregistrées à partir d'un enregistreur numérique avant d'être transcrites. Le projet a été approuvé par le Comité d'éthique de la recherche avec les êtres humains du Réseau de santé Vitalité et le consentement écrit des participants a été obtenu. Les noms utilisés dans cette publication sont fictifs.

\section{Méthode d'analyse des données}

Nous avons dans un premier temps suivi le processus inductif de codification menant à la réduction des données élaboré par Blais et Martineau (2007). Ce processus comporte quatre étapes. Tout d'abord, nous avons préparé les données brutes. Une mise en forme des verbatims (enlever certaines questions qui ne contribuent pas à la compréhension du contexte, restructurer légèrement certaines phrases pour enlever des éléments mineurs qui alourdissent le texte sans ajouter de contenu, regrouper certains propos semblables ainsi qu'éliminer les répétitions) a été effectuée par les chercheurs. Par la suite, nous avons trouvé les unités de sens. Nous avons choisi, comme le recommandent Paillé et Mucchielli (2010), quatre verbatims. Nous avons choisi les verbatims selon certains critères : les entrevues de deux femmes et deux hommes et venant de participants qui avaient des propos variés. Cela a permis une première familiarisation avec le corpus pour les chercheurs qui n'avaient pas participé à la collecte de donnée. Les verbatims choisis étaient tous en français. Nous avons procédé à une lecture attentive et approfondie de ces textes afin de dégager le sens global de l'expérience vécue des participants. Un des chercheurs a fait des résumés afin de dégager des unités de sens. Les deux autres ont identifié, de manière indépendante et directement à partir des verbatims retravaillés, des segments de textes signifiants (unité de sens) et y ont associé un mot-clé. La troisième étape de ce processus est l'identification et la description des premières catégories. À partir des unités de sens, nous avons dégagé, ensemble, les catégories émergentes liées aux objectifs de recherche. Ceci a impliqué une réorganisation de ces catégories en sous-catégories et en catégories plus générales. Cette codification a été effectuée à l'aide du logiciel NVivo 10. Finalement, la dernière étape a été de réviser le raffinement des catégories. Une fois la liste des catégories établie, l'ensemble des verbatims a été lu par un des chercheurs afin de vérifier s'il avait de nouvelles catégories. Comme les chercheurs étaient aussi à l'aise en français qu'en anglais, les verbatim anglais n'ont pas été traduits et ont été traités de la même façon que les français.

Ce que nous présentons ici n'est pas l'ensemble des catégories, mais un certain nombre de catégories qui nous sont apparues signifiantes.

\section{Résultats}

Plusieurs thèmes sont ressortis lors de l'analyse de nos entretiens avec les individus recevant des traitements pour le cancer. Les thèmes généraux, soit les intentions d'agir, les agirs nouveaux et le maintien ou l'intention de maintien des agirs antérieurs, nous ont permis de voir émerger plusieurs sous-thèmes.

Les intentions d'agir ou de maintenir les agirs antérieurs

Intentions d'agir

Rappelons que l'intention d'agir se traduit par le fait d'avoir un objectif à atteindre (Assagioli, 1989). Nos résultats indiquent que les participants avaient des objectifs, des buts fixés pour leur futur. La plupart avaient l'intention d'effectuer des changements à leur quotidien suite aux traitements. Avoir l'intention d'être plus attentifs à soi-même et aux autres sont deux des sous-thèmes qui ont émergé de nos résultats. Les participants ont 


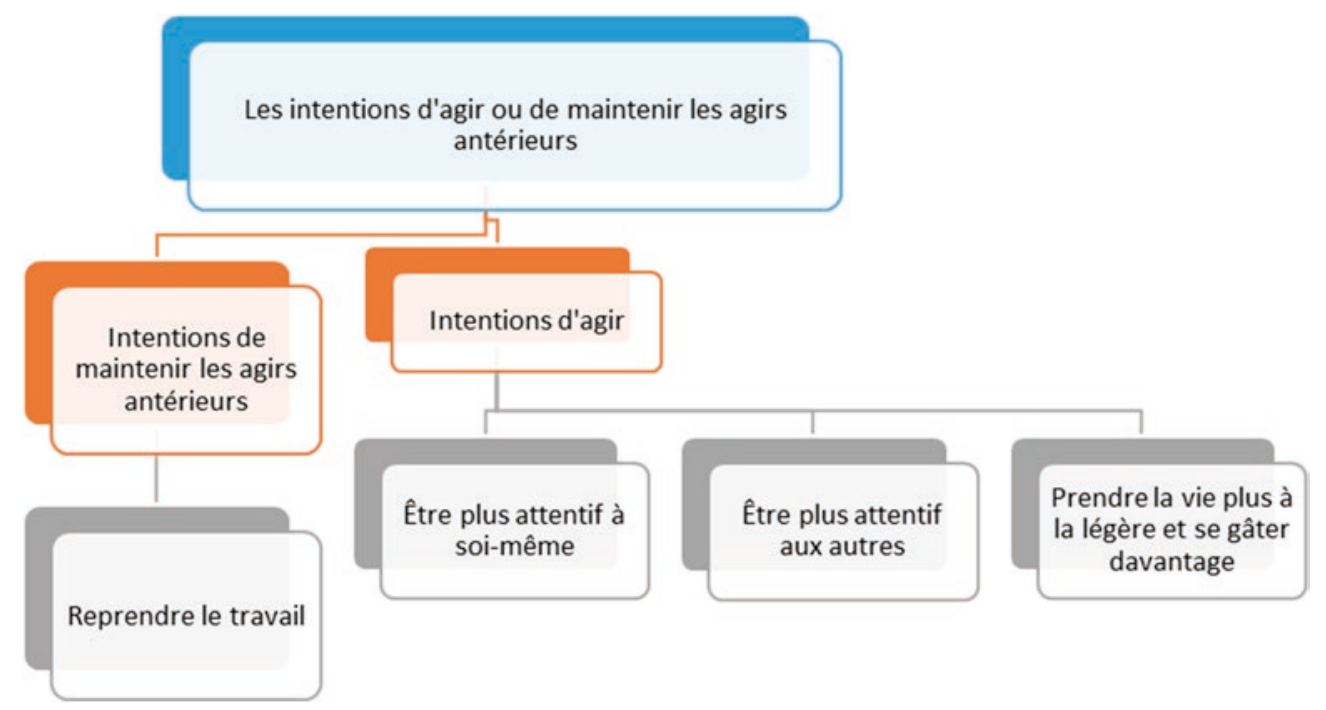

Figure 1: Les intentions d'agir ou de maintenir les agirs antérieurs

aussi exprimé leur volonté de prendre la vie plus à la légère et de se gâter davantage (Figure 1).

Être plus attentif à soi-même Certains participants ont mentionné qu'ils avaient l'intention, lorsque leurs traitements seraient terminés et qu'ils seraient de retour chez eux, d'être plus attentifs à eux-mêmes et à leurs besoins :

\section{Disons que je vais peut-être plus penser à moi. C'est parce que moi, je fais beaucoup de bénévolat et je pense beaucoup aux autres. Je vais être plus attentive à ce que moi i'ai besoin. C'est mon plan ... (Irène)}

Le fait de penser à eux-mêmes en se mettant moins de pression au travail sont des résolutions que certains participants ont mentionnées. Un des participants évoque :

\begin{abstract}
Il faut que je me modère absolument malgré que j'aime ça. Il faut absolument que je délaisse ça, mon travail. En faire un petit peu, mais ne pas pousser seulement pour satisfaire les clients. Après un bout, tu vas t'apercevoir, il faut que tu penses à toi-même. Si c'est pour aller en voyage ou aller à la pêche... (Joseph).
\end{abstract}

Afin de prendre soin d'elle, une des participantes souligne qu'elle a l'intention d'aller à des rendez-vous avec son médecin plus souvent :

\begin{abstract}
Je vais aller pour des tests et des choses comme ça, parce qu'avant ça, i'y allais à tous les 4-5 ans parce que ce n'est pas quelque chose qui est vraiment plaisant. Maintenant, quand ils me diront qu'il faut que i'y aille, j'irai » (Yvonne).
\end{abstract}

Les participants indiquent qu'ils ont l'intention de dire " non » davantage lorsqu'ils auront des demandes provenant de la communauté ou encore de l'entourage. Ils souhaitent établir des priorités afin d'être plus attentifs à leurs propres besoins et demeurer en santé. Une participante explique :
Je pense que je vais plus penser à moi, après ça, ce sera les autres. Je vais dire non plus facilement, parce que c'était toujours oui, même si ça ne me tentait pas de le faire (Irène).

Etre plus attentif aux autres (particulièrement aux autres personnes atteintes de cancer) Même si cela peut sembler en contradiction avec les résultats précédents, plusieurs participants expliquent qu'ils veulent tenter d'être plus attentifs aux besoins des autres. Par exemple, une des participantes explique :

Quand quelqu'un aura le cancer, je vais être plus attentive, l'aider, lui dire « il y a tout le temps de l'espoir, ça va bien aller après », mais ça dépend aussi de la personne. Tu sais, tu ne peux pas être positive avec quelqu'un qui a un cancer à répétition (Irène).

Pour Madeleine, l'intention est de démonter une attitude plus positive afin d'aider les autres à faire de même :

Je vais changer ma façon de voir les choses. Peutê̂tre être plus attentive et rendre les autres heureux aussi. Plus heureuse, plus de gentillesse, encourager les autres, tu sais, supporter pour remonter le moral.

Prendre la vie plus à la légère et se gâter davantage Après leurs traitements, les participants avaient l'intention de prendre la vie plus à la légère en faisant moins d'activités ou de tâches qui les ennuient et aussi de se gâter davantage. Une participante exprime : " Je vais essayer d'être plus coquette. Je vais essayer d'aller plus à la danse et faire des choses pour moi » (Yvonne). Les résultats indiquent également que les participants ne veulent pas attendre à plus tard pour faire des choses qu'ils aiment. Un participant mentionne « J'ai changé ma façon de voir la vie. Avant ça on essayait de ramasser de l'argent pour l'avenir, mais là je vais retirer mes REER, on va profiter de la vie » (Henry). 


\section{Intentions de maintien de certains agirs antérieurs à la maladie}

Le retour à la vie quotidienne suite aux traitements est inévitable. Beaucoup d'agirs antérieurs, qu'ils soient positifs ou négatifs, sont des points d'ancrage dans le quotidien. Toutefois, le seul qui se soit démarqué chez les participants de notre étude est celui de reprendre le travail.

Reprendre le travail Plusieurs participants, malgré les nombreux changements qu'ils ont l'intention d'adopter, comptent tout de même reprendre les activités qu'ils avaient avant leur cancer et avant leurs traitements. Une participante exprime vouloir reprendre le travail lorsque ses traitements seront terminés:

Je parle à ma fille et je parle aux enfants et je reste positive que ça va guérir et que je vais $\mathrm{m}^{\prime}$ en aller. Je vais retourner travailler ce printemps. II ne reste plus qu'un traitement à aller, je prends courage. II reste plus qu'un traitement à aller et après c'est fini (Yvonne).

\section{Agirs nouveaux et maintien de certains agirs antérieurs}

Nos résultats ont démontré que les gens atteints de cancer n'ont pas seulement des intentions d'agir, mais qu'ils adoptent aussi de nouveaux comportements, des agirs nouveaux, et ce avant même la fin de leurs traitements. On remarque que certains changements émanent des individus alors que d'autres sont recommandés par les médecins. Certains participants affirment toutefois maintenir leurs agirs antérieurs (Figure 2).

\section{Agirs nouveaux}

Agirs nouveaux émanant des participants Il est ressorti de nos entretiens que le fait de faire face à une maladie comme le cancer amène souvent les patients à adopter certains changements qu'ils jugent bénéfiques pour eux-mêmes. Demander de l'aide à son entourage, prendre du temps pour soi et améliorer ses habitudes alimentaires sont des changements que les participants ont affirmé avoir adoptés de leur propre chef, pendant leurs traitements.

Demander de l'aide à son entourage Le fait de demander de l'aide s'avère difficile pour bien des gens. Certains participants mentionnent qu'ils ont dû apprendre à demander de l'aide à leur entourage, car ils ne sont plus aptes à faire tout ce qu'ils faisaient avant la maladie ou avant de débuter les traitements. Une participante explique :

Le plus difficile, c'est apprendre à demander. Parce qu'on a toujours donné, on a toujours fait nousmême. C'est apprendre... Comme là, je descends les fins de semaines, ma fille fait toujours des petits plats pour les fins de semaine. J'ai toujours quelque chose de fait (Madeleine).
Prendre du temps pour soi La plupart des participants ont mentionné qu'ils prenaient plus de temps pour euxmêmes. Une des participantes souligne l'importance que le temps qu'elle prend pour elle-même soit de qualité. Elle explique :

J'ai ralenti parce que j'étais pas mal... Je me pensais encore à 20 ans. Quelqu'un a besoin de quelque chose, je vais y aller... Je prenais très peu de temps pour moi. Le temps que je prenais pour moi, j'étais fatiguée. J'en prenais, mais en faisant quelque chose quand même (Madeleine).

Une autre participante explique qu'elle prend maintenant plus de temps pour faire des choses qu'elle aime :
J'adore la nourriture. Je prends plaisir à faire la cuisine, n'importe quoi, je trouve les recettes (...) Je regarde les réseaux de télévision de cuisine, j'aime essayer de nouvelles choses. Avant, je n'avais pas le temps de cuisiner de bons repas, car je travaillais beaucoup. Maintenant, et même durant mes traite- ments de chimio, je passe du temps dans la cuisine et je prends le temps de cuisiner (Rachel, traduit de l'anglais par les auteurs).

Améliorer ses habitudes alimentaires Certains participants mentionnent avoir changé leurs habitudes alimentaires pour le mieux. Un participant explique:

Oui, c'est un gros changement : manger différemment. J'ai toujours essayé de bien manger, mais le plus gros changement, je crois, est de manger moins de viande rouge. J'aime beaucoup la viande rouge (...) Nous essayons de manger sainement, mieux qu'avant. Nous avions l'habitude de manger $\mathrm{n}^{\prime}$ importe quoi (Eddy, traduit de l'anglais par les auteurs).

Agirs nouveaux recommandés par les médecins Certains changements adoptés par les personnes atteintes du cancer n'émanent pas des participants mêmes. Les agirs nouveaux peuvent être recommandés par le personnel médical, le plus souvent par les médecins. Ces changements sont surtout liés à la pratique d'activités physique, au repos et à l'alimentation.

Faire plus d'activité physique Les médecins soulignent souvent l'importance de l'activité physique aux patients atteints de cancer. Une participante explique qu'elle fait plus d'exercice suite à une recommandation de son médecin :
«À peu près le seul exercice que je peux faire, c'est marcher, parce que $j^{\prime}$ ai trop mal dans les reins pour faire d'autre exercice, donc je marche autant que je peux. Mon médecin m'a recommandé de marcher » (Yvonne).

Se reposer davantage Les médecins conseillent aussi à plusieurs patients de se reposer davantage. C'est le cas d'un participant qui mentionne : «Ils (les médecins) m'ont dit de me reposer. J'ai dû apprendre à le faire parce que ce 


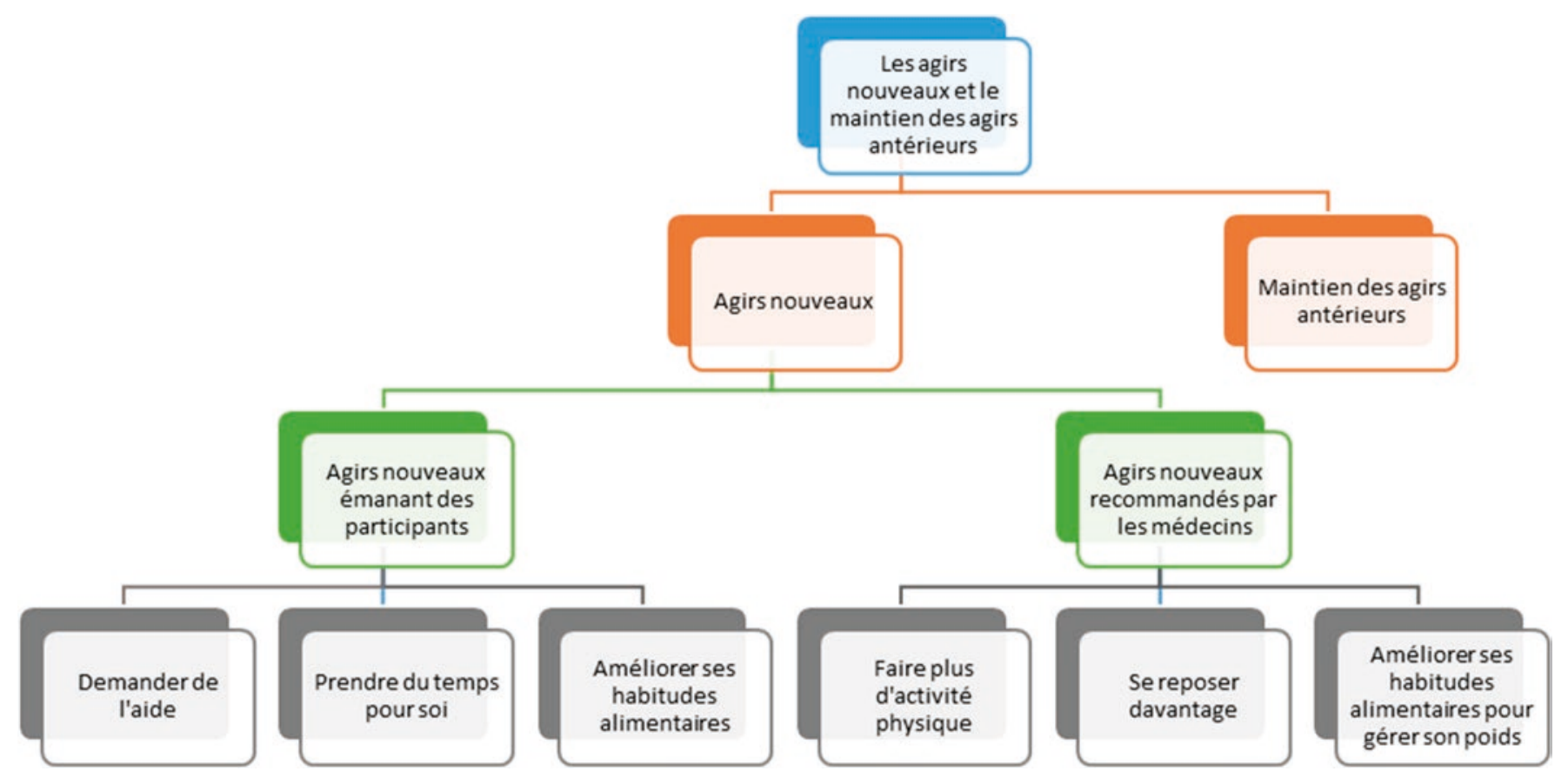

Figure 2: Les agirs nouveaux et le maintien des agirs antérieurs

n'est pas naturel pour moi » (Eddy, traduit de l'anglais par les auteurs).

Améliorer ses habitudes alimentaires pour gérer son poids Une des participante explique que le médecin lui recommande de surveiller ses habitudes alimentaires, car elle ne doit pas perdre trop de poids; elle mentionne " Je surveille ce que je mange parce qu'ils ne veulent pas que je maigrisse trop » (Yvonne). Un autre participant souligne que le médecin lui suggère de perdre un peu de poids et de manger plus sainement :

\begin{abstract}
Mon médecin m'a dit Il faut que tu perdes de la bedaine. Pour te ramener, il faut que tu perdes de la bedaine. C'est ok quand je suis à la maison les fins de semaine, je peux le faire, mais quand tu t'en vas au loin, à quelque part, dans les restaurants, c'est plus difficile (Roland).
\end{abstract}

Maintien de certains agirs antérieurs à la maladie Nos résultats indiquent que la plupart des participants adoptent des changements dans leurs habitudes de vie ou qu'ils ont l'intention d'en adopter. Par contre, certains participants ont souligné qu'ils n'ont adopté aucun changement dans leur vie et qu'ils n'ont aucune intention d'en adopter (Figure 2). Il faut toutefois rappeler que les entrevues ont été conduites en deux fois et que certains participants qui avaient affirmé ne faire aucun changement lors de la première entrevue ont affirmé en faire lors de la deuxième.

Plusieurs mentionnent qu'ils ont toujours bien mangé, et qu'ils n'ont donc pas besoin de changer leurs habitudes alimentaires. Un participant indique « Je mange comme avant. Il n'y a pas rien qui va changer. C'est juste quelque chose qui est arrivé, il n'y a rien qui a changé. Je passe la balayeuse pareille, j'ai mes tâches à faire » (Georges).

D'autres soulignent cependant que si les médecins leur suggéraient d'adopter des agirs nouveaux, ils prendraient ces changements en considération. Ils n'ont toutefois pas l'intention de changer par eux-mêmes :

\section{Non, je crois que j'ai déjà une bonne attitude envers la vie et envers ma santé. Donc, à part si les docteurs me demandent de changer ma diète ou quelque chose, c'est certain que je le ferai, mais à part ça, non. Je ne crois pas qu'il y a quelque chose que je dois changer. (Rachel, traduit de l'anglais par les auteurs)}

\section{Discussion}

Le but de notre recherche était de connaître les occasions de changements dans l'expérience du cancer chez les personnes âgées de 60 ans et plus subissant des traitements pour le cancer. Le hasard a fait que notre participant le plus âgé avait 73 ans. Notre recherche a donc porté sur l'expérience de « jeunes personnes âgées ». Nous avons voulu comprendre la présence ou l'absence d'intentions d'agir et d'agirs nouveaux chez ces personnes. Certaines études ont été faites sur l'expérience des gens atteints de cancer, mais rares sont celles qui ont ciblé directement ce groupe d'âge et les personnes pendant leurs traitements. L'originalité de notre recherche se situe également dans le regard que nous portons non seulement sur les agirs nouveaux, 
qui s'apparentent aux stratégies d'adaptation décrites par d'autres auteurs (ex : Towsley et al., 2007) mais aussi sur les intentions d'agir et le maintien de certains agirs antérieurs à la maladie.

Du point de vue des intentions, nous avons identifié une seule intention de maintenir les agirs antérieurs, soit celle de reprendre le travail après les traitements. Les intentions d'agir dans le futur, quant à elles, se déclinaient en trois thèmes : être plus attentif à soimême, être plus attentif aux autres et prendre la vie plus à la légère tout en se gâtant davantage. Du point de vue des agirs nouveaux déjà en place dans le contexte de la maladie, nous avons pu distinguer des agirs émanant des participants: demander de l'aide, prendre du temps pour soi et améliorer ses habitudes alimentaires. D'autres agirs mis en place par les participants étaient plutôt recommandés par les médecins : faire plus d'activité physique, se reposer davantage et améliorer ses habitudes alimentaires pour gérer son poids. Une minorité de participants a toutefois affirmé n'avoir effectué aucun changement et n'avoir aucune intention en ce sens.

\section{Intentions d'agir}

Nos résultats nous indiquent que la plupart des participants avaient l'intention d'adopter des agirs nouveaux à la suite de leurs traitements. Ces résultats soutiennent les résultats de recherches précédentes, notamment ceux de Senden et al. (2015) et Rabin \& Pinto (2005) qui soulignent que les gens en traitement pour un cancer manifestent souvent des intentions, un désir de changer certaines habitudes de vie.

Les intentions d'agir les plus fréquemment mentionnées par les participants étaient de devenir plus attentifs à soi-même, plus attentifs aux autres (surtout aux autres personnes atteintes de cancer), celle de prendre la vie plus à la légère et de se gâter davantage. Les intentions " être plus attentif à soi » et " être plus attentif aux autres » peuvent a priori sembler en contradiction, mais on remarque que les participants souhaitaient avant tout se délester de certaines tâches physiquement exigeantes ou demandant des déplacements afin d'être davantage présents moralement pour leur entourage. Les intentions d'être plus attentifs à soi, de prendre la vie plus à la légère et de se gâter davantage ont été peu soulevées dans les études sur l'expérience du cancer, même si plusieurs recherches ont souligné le fait que l'expérience du cancer changeait globalement la perspective sur la vie (ex : Halldorsdottir \& Hamrin, 1996; Esteves, Roxo, \& da Conceicao Saraiva, 2015). Il est possible que nos résultats se distinguent de ceux de plusieurs études en raison du fait que nous avons porté attention aux intentions alors que la majorités des études se sont concentrées sur les stratégies et les changements déjà adoptés. Esbensen, Swane, Hallberg, et Thomé (2008) ont toutefois trouvé un résultat semblable au nôtre dans une étude phénoménologique conduite auprès de patients de 65 ans et plus où plusieurs participants ont affirmé vouloir davantage profiter de la vie, malgré les limites imposées par la maladie. Krumwiede \& Krumwiede (2012) ont également trouvé dans leur étude que les hommes âgés atteints du cancer de la prostate souhaitaient profiter davantage de la vie en faisant des activités qui leurs plaisaient mais aussi en aidant davantage les autres, ce qui rejoint également nos résultats.

\section{Les agirs nouveaux}

Les agirs nouveaux sont ceux qui ont été adoptés par les participants pendant leurs traitements. Il est apparu dans nos résultats que certains agirs émanaient des individus eux-mêmes (demander de l'aide, prendre du temps pour soi et améliorer ses habitudes alimentaires) alors que d'autres étaient recommandés par le personnel médical (faire plus d'activité physique, se reposer davantage et améliorer ses habitudes alimentaires pour gérer son poids). Nous n'avons toutefois pas pu, comme l'ont fait Doyon et al. (2008) dans leur recherche menée auprès d'enseignants, discerner des agirs spontanés et des agirs délibérés. Il est possible que le contexte de la maladie (une annonce souvent inattendue et des traitements qui débutent rapidement) précipite les individus vers des agirs possiblement nécessaires à la survie, ou du moins à l'amélioration immédiate de la qualité de vie.

L'agir nouveau de " demander de l'aide » a également été identifié en tant que stratégie d'adaptation par Fredette (1995) et Wengstrom, Haggmark, et Forsberg (2001) dans des études menées auprès de femmes ayant survécu au cancer du sein. Le fait de prendre du temps pour soi a été identifié par Thibodeau et MacRae (1997) également auprès de femmes ayant reçu un diagnostic de cancer du sein. Les études qui ont trouvé que les gens atteints de cancer voulaient faire davantage d'activités qu'ils aiment (Esbensen et al., 2008; Krumwiede \& Krumwiede, 2012) vont également dans le sens de « prendre du temps pour soi ».

Nos résultats indiquent que certains participants optaient pour des changements au niveau alimentaire tout comme Hopkinson (2007) l'a souligné dans son étude. Senden et al. (2015) ont, pour leur part, souligné que les tâches quotidiennes étaient différentes de celles effectuées avant le diagnostic du cancer. Le fait que notre groupe de participants séjournait hors de leurs domiciles durant leurs traitements implique que leur quotidien était forcément différent. Il était ainsi difficile de distinguer ceci dans nos résultats. 
Il est intéressant de noter qu'aucun participant n'a affirmé avoir ou vouloir effectuer des changements qui auraient été recommandés par leur entourage, même si la question leur a été posée explicitement pendant les entrevues et que tous bénéficiaient du soutien de leur entourage. Nous avions aussi demandé aux participants s'ils avaient cherché à obtenir de l'information (médias, ateliers, lectures...) sur le cancer et sur des changements qu'ils pourraient effectuer afin d'améliorer leurs chances de guérison et possiblement diminuer les chances de récidives. Tous ont répondu par la négative. Ces résultats sont en contradiction avec les résultats obtenus par Fredette (1995) qui a identifié la recherche $d$ 'information comme étant une stratégie d'adaptation adoptée par des femmes ayant eu un diagnostic de cancer du sein. Il est possible que leur niveau de scolarité explique en bonne partie ces résultats. Plusieurs auteurs (Van der Heide et al., 2013; Morris et al., 2013) ont liés un faible niveau d'éducation à un faible niveau de littératie en santé et, par conséquent, à une capacité réduite à obtenir et comprendre des informations liées à la santé. Towsley et al. (2007) avaient d'ailleurs noté que des facteurs sociaux et financiers influençaient (positivement ou négativement) les stratégies d'adaptation utilisées. Les agirs nouveaux recommandés par le personnel médical semblaient toutefois sérieusement pris en considération par les participants. Selon les résultats obtenus par Morris et al. (2013) dans une étude conduite auprès de 1013 adultes de 40 à 70 ans, les adultes ayant de faibles niveaux de littératie étaient davantage portés à obtenir de l'information sur la santé de la part des professionnels de la santé que de toute autre source, incluant la famille, les amis et l'internet. Ces résultats vont dans le sens de ceux que nous avons obtenus.

\section{Intentions de maintien et maintien des agirs antérieurs à la maladie}

Tel que mentionné plus tôt, certains participants qui avaient affirmé maintenir ou avoir l'intention de maintenir certains agirs antérieurs à la maladie lors de la première entrevue se sont contredits lors de la deuxième entrevue. Il est fort possible que la première entrevue ait agit comme point de départ à une réflexion qui aura mené à des agirs nouveaux ou des intentions d'agir au cours des semaines suivantes. Il est également possible que des participants aient réalisé après l'entrevue qu'ils avaient tout de même effectué certains changements subtils dans leur vie, ou encore qu'ils aient voulu plaire au chercheur. Certains participants ont toutefois maintenu qu'ils n'avaient effectué aucun changement. Il faut considérer que, dû à leurs types de cancer, la plupart des participants avaient peu de douleur physique et peu d'effets secondaires de leurs traitements. Ainsi, les participants n'ont pas mentionné l'expérience des traitements en tant que telle comme ayant eu un impact sur leurs agirs et intentions d'agir. Certains avaient même de la difficulté à croire qu'ils étaient malades. Cela est particulièrement vrai pour les hommes atteints du cancer de la prostate. Ce type de cancer évolue très lentement et le taux de survie après 5 ans est de 95\% (Société canadienne du cancer, 2016). Dans ce contexte, la maladie altère moins la qualité de vie et il est possible que les patients soient moins portés à effectuer des changements, ce qui rejoint les résultats obtenus par Thomé et al. (2004). Hopkinson (2007) remarque que ce choix de ne pas effectuer de changement peut aussi être influencé par l'entourage de la personne. Certaines circonstances dans la vie de la personne atteinte peuvent par ailleurs entraver et affecter l'auto-efficacité. L'auteure explique donc que le fait de changer d'environnement ou de ressources personnelles pourrait aider à faciliter la présence de changements positifs.

Certains participants ont manifesté explicitement leur intention de retourner à certains agirs antérieurs à la maladie en retournant au travail. Il y a certainement quelque chose de rassurant dans l'idée de retourner à ses habitudes dans un contexte où la maladie contrôle une grande partie de la vie. Fredette (1995), Wengstrom, Haggmark, and Forsberg (2001), Towsley et al. (2007), et Senden et al. (2015) avaient également identifié la poursuite du travail comme étant une stratégie d'adaptation s'inscrivant dans une volonté de retour à la routine habituelle.

Towsley et al. (2007) soulignaient, dans leur étude, que plusieurs personnes choisissaient de maintenir leurs anciennes habitudes et de n'adopter aucun changement. Nos résultats appuient cette étude, mais nous permettent également de souligner que la raison principale pour laquelle certains participants optaient pour le maintien des agirs antérieurs à la maladie est qu'ils croyaient sincèrement ne rien devoir changer. Nous avons remarqué que plusieurs participants affirmaient qu'ils avaient déjà de bonnes habitudes de vie, ne croyaient pas que ces dernières pouvaient être liées à leur maladie et ne voyaient donc pas la nécessité d'effectuer des changements.

\section{Conclusion}

Cette recherche nous a permis de mettre en relief l'expérience du cancer comme occasion de changement chez des gens âgés de 60 à 73 ans en traitement pour un cancer et séjournant dans une auberge qui accueille des patients en traitements oncologiques. Il est toutefois à noter que notre étude ne couvre pas toute la diversité des cancers et des expériences engendrées par chacun. L'expérience de vivre un cancer chez soi, possiblement dans la solitude, sans le support du personnel et des patients de l'auberge, n'est pas non plus reflétée. 
L'expérience de gens plus âgées que nos participants pourrait aussi être différente en raison de contraintes de santé supplémentaires et de contexte sociaux possiblement différents (davantage de veufs, moins de temps dédié au travail et au bénévolat, résidence en foyers de soins, etc.).

Notre approche originale du sujet nous a permis de poser un regard nouveau sur la façon dont les individus plus âgés effectuent ou planifient effectuer des changements dans leur vie dans le contexte de la maladie. Nos résultats mettent en relief le fait que la majorité des participants ont manifesté des intentions d'agir ou ont adopté de nouveaux agirs pendant la période où ils recevaient des traitements en oncologie. Nous avons toutefois noté que les agirs et intentions d'agir identifiés chez nos participants différaient de ceux identifiés dans une majorité d'études sur l'expérience du cancer en raison du fait qu'ils étaient surtout centrés sur le vécu relationnel et les activités des participants plutôt que sur la modification des habitudes de vie. Dans la plupart des cas, lorsque des modifications avaient été apportées aux habitudes de vie, elles avaient été recommandées par un médecin. Les participants ont en effet été très attentifs aux recommandations des médecins, ce qui souligne l'importance de ces derniers non seulement dans l'offre des traitements mais aussi comme conseillers. L'âge des participants pourrait expliquer en partie la difficulté à envisager de modifier des habitudes de vie souvent bien ancrées. Toutefois, on peut également supposer que la logistique ou les coûts qui peuvent $s^{\prime}$ associer à la modification de certaines habitudes de vie peuvent aussi avoir agi comme frein. Plusieurs participants ne semblaient toutefois pas établir de liens entre les habitudes de vie et la maladie. Le niveau de scolarité pourrait ici avoir joué un rôle en les rendant moins enclins à chercher par eux-mêmes de l'information sur le cancer ou encore sur des façons de contribuer à la guérison ou de diminuer les chances de récidives. Il est également possible que les habitudes de vie de nos participants étaient déjà adéquates et qu'ils n'aient donc pas vu la nécessiter de s'informer sur le sujet. Il est aussi intéressant de noter que l'entourage avait très peu d'influence sur les choix, et ce même si tous les participants, en couple ou non, semblaient bénéficier d'un bon réseau familial et social. Les participants se sont montrés fermés à l'idée de recevoir des conseils de leur entourage. Il faut dire qu'ils avaient pour la plupart déjà été confrontés à des situations de santé difficiles dans le passé et semblaient avoir déjà développé leur propre façon de gérer la maladie. Ils ne semblaient donc pas en quête de solutions extérieures.

Les participants ont partagé avec beaucoup de détails les impacts du cancer sur leur vie quotidienne mais il nous a été difficile d'obtenir de leur part une signification attribuée à la maladie et aux choix effectués, malgré les questions posées en ce sens. Il est possible que nos participants aient trouvé difficile de partager cet aspect de la maladie avec la chercheure. Nous avons toutefois plutôt l'impression que la plupart d'entre eux ont préféré considérer la maladie comme une malchance entrainant la modification de certains aspects de leur vie, sans toutefois entraîner une quête de sens.

Si on considère que la maladie peut être une occasion d'apprentissage et de changement, le cadre de Doyon et al. (2008), qui examine les processus de transformation intérieur sous l'angle des intentions d'agir et des agirs nouveaux, s'avère fertile pour la conduite de nombreuses recherches auprès des patients. Il serait ainsi intéressant de poursuivre l'exploration de l'expérience du cancer chez les gens âgés qui ne séjournent pas dans une auberge spécialisée pendant la durée des traitements, et aussi auprès d'un groupe d'individus plus scolarisé afin d'explorer les différences potentielles en ce qui concerne le cancer comme occasion de changement. Il serait également intéressant d'effectuer des entrevues quelques mois après le traitement afin de comprendre de quelle façon les intentions d'agir et les agirs nouveaux évoluent dans le temps chez les gens âgés. Ces résultats devraient aussi mener à une réflexion sur la prise en considération des particularités des personnes âgées peu scolarisées dans les soins et l'accompagnement qui leurs sont offerts dans le contexte de la maladie. Le rôle prépondérant des médecins dans l'émergence d'agirs nouveaux chez ces patients devrait inciter les médecins à s'engager dans un dialogue avec leurs patients afin de comprendre comment la maladie influence leurs relations, leurs activités et leurs habitudes de vie et ainsi leur offrir des conseils ou les diriger vers des ressources adaptées à leur réalité.

\section{References}

Andrykowski, M., Brady, M., \& Hunt, J. (1993). Positive psychosocial adjustment in potential bone marrow transplants: Cancer as a psychosocial transition. Psycho-oncology, 2, 261-276.

Arden Pope III, C., Burnett, R., Thun, M., Calle, E., Krewski, D., Ito, K., \& Thurston, G. (2002). Lung cancer, cardiopulmonary mortality and long-term exposure to fine particulate air pollution. Journal of the American Medical Association, 287(9), 1132-1141.

Assagioli, R. (1989). La volonté libératrice. Paris: Le Hierach.

Barbaric, M., Brookes, E., Moore, L., \& Cheifetz, O. (2010). Effects of physical activity on cancer survival: A systematic review. Physiotherapy Canada, 62(1), 25-34.

Blais, M., et Martineau, S. (2007). L'analyse inductive générale: description d'une démarche visant à donner 
un sens à des données brutes. Recherches qualitatives, 26(2), 1-18.

Blanchard, C. L., Tanenbaum, S., \& Hidy, G. M. (2014). Spatial and temporal variability of air pollution in Birmingham, Alabama. Atmospheric Environment, 89, 382-391.

Cordova, M., \& Andrykowski, M. (2003). Responses to cancer diagnosis and treatment: Posttraumatic stress and posttraumatic growth. Seminars in Clinical Neuropsychiatry, 8(4), 286-296.

Doraia, T., \& Aggarwal, B. (2004). Role of chemopreventive agents in cancer therapy. Cancer Letters, 215, 129-140.

Doyon, A., Legault, M., et Paré, A. (2008). Analyse de pratique et processus de transformation chez des éducateurs. Nouveau cahier de la recherche en éducation, 11(1), 17-34.

Esbensen, B. A., Swane, C., Hallberg, I., \& Thomé, B. (2008). Being given a cancer diagnosis in old age: A phenomenological study. International Journal of Nursing Studies, 45, 393-405.

Esteves, A., Roxo, J., \& da Conceicao Saraiva, M. (2015). The lived experience of people with progressive advanced cancer. British Journal of Nursing, 24(10), 15-21.

Fredette, S. (1995). Breast cancer survivors: Concerns and coping. Cancer Nursing, 18, 35-46.

Fromm, K., Andrykowski, M., \& Hunt, J. (1996). Positive and negative psychosocial sequelae of bone marrow transplantation: Implications for quality of life assessment. Journal of Behavioral Medicine, 19(3), 221-240.

Garneau, J., et Larivey, M. (1979). L'auto-développement. Montréal: Les Éditions de 1'Homme.

Halldorsdottir, S., \& Hamrin, E. (1996). Experiencing existential changes: The lived experience of having cancer. Cancer Nursing, 19(1), 29-36.

Heidermann, C., Schulze, C., Franco, O., van Damm, R., Mantzoros, C., \& Hu, F. (2008). Dietary patterns and risk of mortality from cardiovascular disease, cancer and all causes in a prospective cohort of women. Circulation, $118,230-237$.

Hopkinson, J. (2007). How people with advanced cancer manage changing eating habits. Journal of Advanced Nursing , 59(5), 454-462.

Karsenti, T., et Savoie-Zajc, L. (2011). La recherche en éducation: Étapes et approches (3e ed.). Montréal: ERPI.

Komura, K., \& Hegarty, J. (2006). The appraisal of positive life changes following cancer diagnosis: An interview study. Palliative E Supportive Care, 4(1), 3-12.

Krukowski, R., \& Smith West, D. (2010). Consideration of the food environment in cancer risk reduction. Journal of the American Dietetic Association, 110, 842-844.
Krumwiede, K., \& Krumwiede, N. (2012). The lived experience of men diagnosed with prostate cancer. Oncology Nursing Forum, 39(5), 443-450.

Morris, N., Field, T., Wagner, J., Cutrona, S., Roblin, D., Gaglio, B. (2013). The association between health literacy and cancer-related attitudes, behaviors and knowledge. Journal of Health Communication, 18, 223-241.

Paillé, P., et Muchielli, A. (2010). L'examen phénoménologique des données empiriques. L'analyse qualitative en sciences sociales et humaines, 2, 85.

Petrie, K., Buick, D., Weinman, J., \& Booth, R. (1999). Positive effects of illness reported by myocardial infraction and breast cancer patients. Journal of Psychosomatic Research, $47(6), 537-543$.

Rabin, C., \& Pinto, B. (2005). Cancer-related beliefs and health behavior change among breast cancer survivors and their first-degree relatives. Psycho-oncology, 15, 344-354.

Senden, C., Vandecasteele, T., Vandenberghe, E., Versluys, K., Piers, R., \& Grypdonck, M. (2015). The interaction between lived experiences of older patients and their family caregivers confronted with a cancer diagnosis and treatment: A qualitative study. International Journal of Nursing Studies, 52, 197-206.

Société américaine du Cancer. (2016). American Cancer Society. Consulté le Juin, 9, 2016, sur American Cancer Society: Available at: www.cancer.org.

Société canadienne du cancer. (2016). Analysis of the impact of population growth and aging on cancer incidence. Récupéré sur Société canadienne du cancer: Available at: http:/ / www. cancer.ca / canada-wide/about $\% 20$ cancer / cancer $\% 20$ statistics / canadian $\% 20$ cancer $\% 20$ statistics /special $\% 20$ topics / analysis $\% 20$ of $\% 20$ the $\% 20$ impact $\% 20$ of $\% 20$ population $\% 20$ growth $\% 20$ and $\% 20$ aging $\% 20$ on $\% 20$ cancer\%20incidence.aspx?sclang=en.

Société canadienne du cancer. (2017). Statistiques canadiennes sur le cancer 2017. Récupéré de : http://www.cancer. $\mathrm{ca} / \sim$ / media/cancer.ca/CW / cancer\%20information/ cancer $\% 20101 /$ Canadian $\% 20$ cancer $\% 20$ statistics / Canadian-Cancer-Statistics-2017-FR.pdf?la=fr-CA.

Statistique Canada. (s.d.). Recensement de 2016. Consulté le 5 avril 2018 sur Statistique Canada: Available at: http: / / www12.statcan.gc.ca/census-recensement/2016/dp$\mathrm{pd} /$ prof/index.cfm?Lang=F.

Thibodeau, J., \& MacRae, J. (1997). Breast cancer survival: A phenomenological inquiry. Advances in Nursing Science, $19,65-74$.

Thomé, B., Esbensen, B., Dykes, A.-K., \& Hallberg, I. (2004). The meaning of having to live with cancer in old age. European Journal of Cancer Care, 13, 399-408.

Towsley, G., Beck, S., \& Watkins, J. (2007). Learning to live with it: Coping with the transition to cancer survivorship in older adults. Journal of Aging Studies, 21, 93-106. 
Van der Heide, I., Wang, J., Droomers, M., Spreeuwenberg, P., Rademakers, J., \& Uiters, E. (2013). The relationship between health, education and health literacy: Results from the Dutch adult literacy and life skills survey. Journal of Health Communication, 18, 172-184.

Van Manen, M. (1997). Researching lived experience: Human science for an action sensitive pedagogy. Toronto, Canada: The Althouse Press.
Vineis, P., Hoek, G., Krzyzanowski, M., Vigna-Taglianti, F., \& Veglia, F. (2007). Lung cancer attribuable to environmental tobacco smoke and air pollution in non-smokers in different European countries: A prospective study. Environmental Health, 6(7), 1-7.

Wengstrom, Y., Haggmark, C., \& Forsberg, C. (2001). Coping with radiation therapy: Strategies used by women with breast cancer. Cancer Nursing, 24(4), 264-271. 